\title{
Polymer-Based Nano/Composites: Theory, Synthesis, Modifications, and Properties
}

\author{
Mircea Chipara, ${ }^{1}$ Alan K. T. Lau, ${ }^{2}$ Mahmood Aliofkhazraei, ${ }^{3}$ \\ Angel Romo-Uribe, ${ }^{4}$ and Ehsan Bafekrpour ${ }^{5}$

\begin{abstract}
${ }^{1}$ Department of Physics and Astronomy, The University of Texas Rio Grande Valley, 1201 W. University Drive, Edinburg, TX 78539, USA

${ }^{2}$ Department of Mechanical Engineering, The Hong Kong Polytechnic University, Hung Hom, Hong Kong

${ }^{4}$ Instituto de Ciencias Físicas, Universidad Nacional Autónoma de México (UNAM), Cuernavaca, MOR, Mexico

${ }^{5}$ Centre for Advanced Materials and Performance Textiles, RMIT University, Room 8, Building 512, 25 Dawson Street,
\end{abstract} \\ ${ }^{3}$ Department of Materials Science, Faculty of Engineering, Tarbiat Modares University, Tehran, Iran \\ Brunswick, VIC 3056, Australia
}

Correspondence should be addressed to Mircea Chipara; chipara@yahoo.com

Received 25 August 2015; Accepted 25 August 2015

Copyright (C) 2015 Mircea Chipara et al. This is an open access article distributed under the Creative Commons Attribution License, which permits unrestricted use, distribution, and reproduction in any medium, provided the original work is properly cited.

The need for better materials has driven us to composites, obtained by combining two or more materials with different physical and chemical properties. The first composite (wattle and daub) was discovered about 6,000 years ago and the first polymer-based composite (Bakelite) was patented in 1907. In polymer-based composites, one or more materials labelled as fillers are dispersed within a polymeric matrix (homopolymer, copolymer, block copolymer, or polymer blend). While it is possible to speculate in more detail about the terminology of polymer-based nanocomposites, the general perception is that a nanosized filler is required.

The nanorevolution opened the door to the fabrication of submicron confined particles. Typically, such particles known as "nanoparticles" have at least one dimension less than $100 \mathrm{~nm}$. Two main techniques, namely, bottom-up and topdown, are competing for the fabrication of nanoparticles. In the bottom-up approach atoms and molecules are organized as nanoparticles while the top-down approach starts for micron or larger particles and reduces their dimensions. Typical bottom-up paths are represented by chemical synthesis, sputtering, electrodeposition, and chemical vapor deposition while among the top-down techniques it is possible to include the energetic mechanical milling and sonication.

The possibility to obtain particles confined at nanometer scale ignited an intense worldwide theoretical and experimental research aiming at synthesizing new nanoparticles and at better understanding their properties. The reasons behind this effort derive from the amazing new or enhanced features of nanoparticles, triggered by the transition from the "classical" behavior to the "quantum behavior." The submicron confinement of matter (of quantum nature) is responsible for important changes such as the shift of electronic energy levels towards higher energies as the size of the confinement is decreased, as observed in quantum wells, where the energy levels $E$ for an electron in an infinite one-dimensional well of width $L$ are $E_{n}=h^{2} n^{2} /\left(8 m L^{2}\right)$, where $m$ is the mass of the electron and $n$ the quantum number associated with the energy level. Another example is the dependence of the energy gap $E_{G}$ of semiconducting nanoparticles on their radius $E_{G}(R)=E_{G}(\infty)+$ $\hbar^{2} \pi^{2} /\left(2 R^{2}\right)\left(1 / m_{e}+1 / m_{h}\right)$, where $E_{G}(\infty)$ is the gap energy for an infinite particle, $m_{e}$ is the electron mass, $m_{h}$ is the hole's mass, $\hbar$ is Planck's constant, $R$ is the radius of the nanoparticle, and $E_{G}(R)$ is the gap energy of a particle of radius $R$.

Surface dominating effects may have important contributions to the physical properties of nanoparticles. However, some surface dominating effects have not a quantum nature being a consequence of the fact that the number of atoms located on the surface of nanoparticles is no more negligible compared to the number of atoms located within the core of the nanoparticles, as it was for micron scale or larger particles. 
Polymer-based nanocomposites are materials obtained by dispersing such nanoparticles within various polymeric matrices such as homopolymers, copolymers, block copolymers, and polymer blends. This includes matrices with a wide variety of physical and chemical properties starting from structural capabilities to high elastic features, from hydrophobicity to hydrophilicity, and from electrical insulating features (polyethylene) to semiconductors or conductors (doped polyaniline, polyacetylenes). An amazing feature of these polymeric matrices is that they are porous, leaving the confined nanoparticle relatively exposed. This has been already speculated in various applications such as polymer deposited catalysts.

The overall physical and chemical properties of polymerbased nanocomposites are controlled by the properties of the nanoparticles, the features of the polymeric matrix, and the interactions between nanoparticles and the polymeric matrices. In polymer-based nanocomposites the huge surface area of the nanoparticles results in a large fraction of polymer being confined within the polymer-nanoparticles interface and eventually in a gradual transition of the physical and chemical properties from "bulk" matrix-controlled features to interphase or "surface" dominated characteristics. A good dispersion of the nanoparticles is mandatory to observe such transition. Typically, the enhanced thermal stability of polymer-based nanocomposites and the shifts of the main phase transitions occurring within the polymeric matrix as a result of the loading with nanoparticles are the main consequences of a thick polymer-nanoparticle interface.

All these competing factors resulted in a huge number of polymer-based nanocomposites with diverse applications, amazing physical and chemical features, and almost infinite possibilities. This issue focuses on various aspects of polymerbased nanocomposites, starting from synthesis and characterization up to applications. Owing to the structure of the polymeric matrix, many emerging applications are expected in the area of biology and medicine. The issue provides a relevant snapshot of the actual status of polymer-based nanocomposites starting from fabrication issues up to final (potential) applications.

Mircea Chipara

Alan K. T. Lau Mahmood Aliofkhazraei Angel Romo-Uribe Ehsan Bafekrpour 

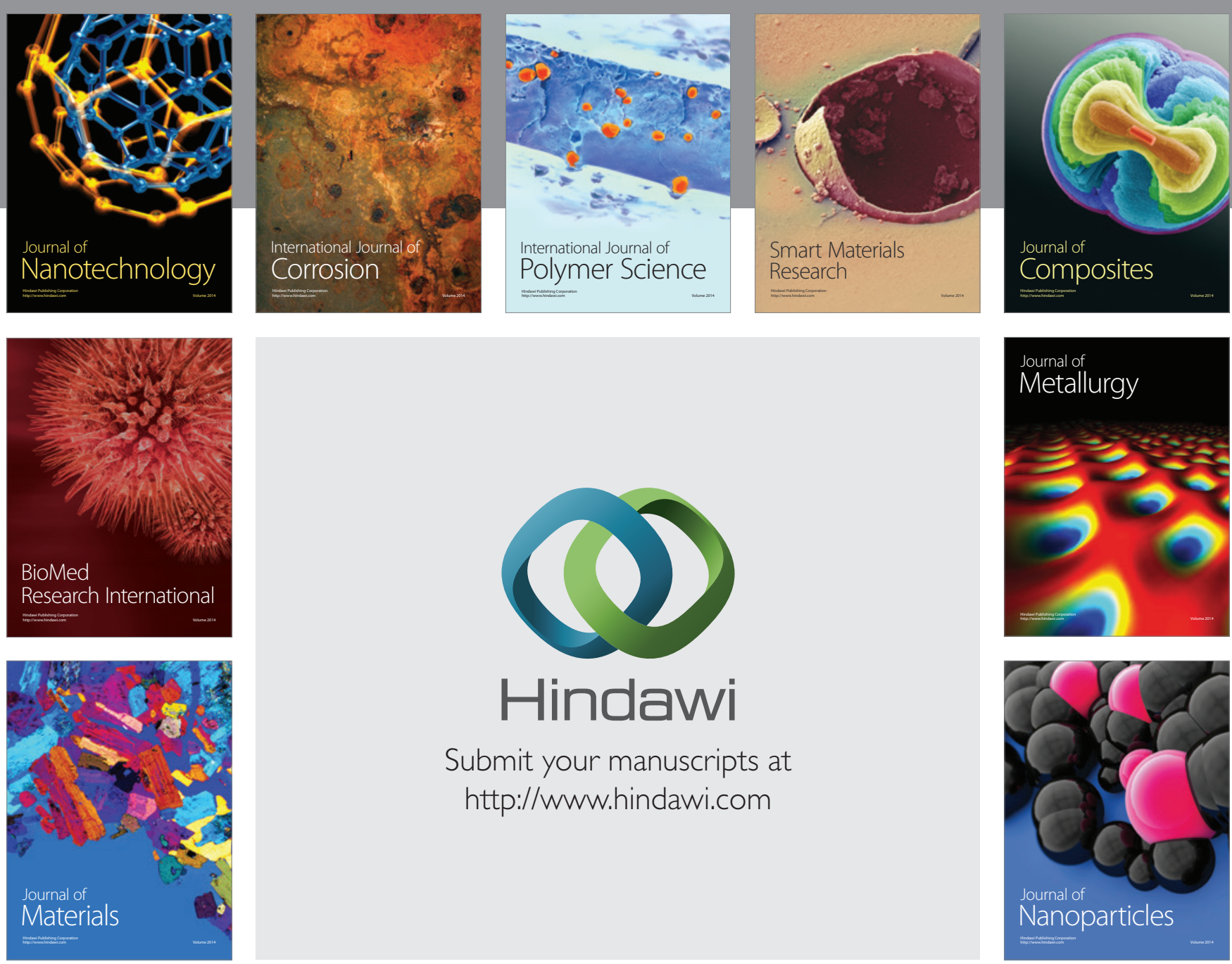

Submit your manuscripts at http://www.hindawi.com
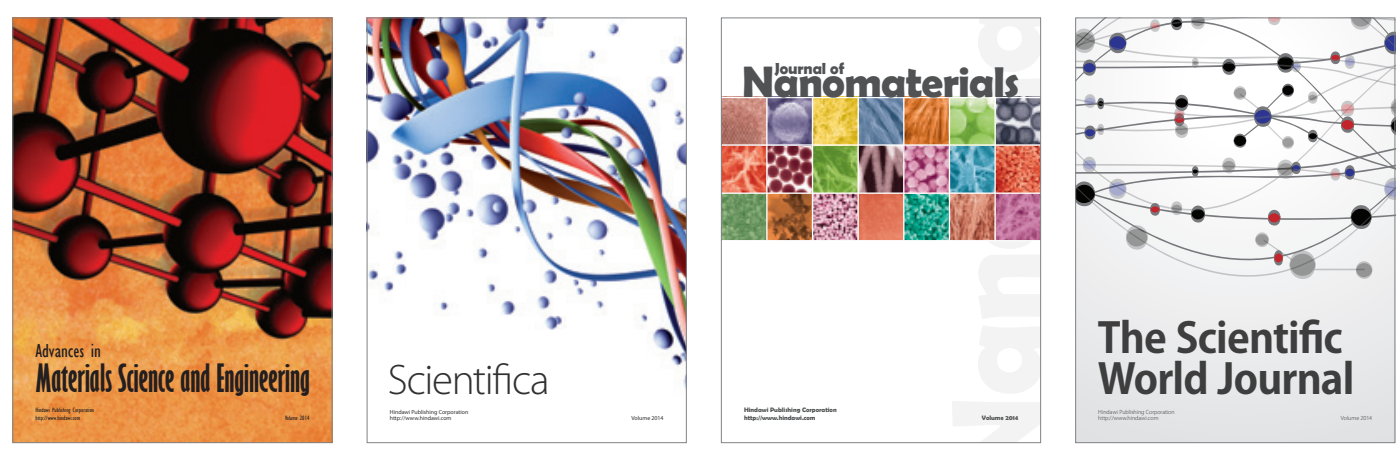

\section{The Scientific World Journal}
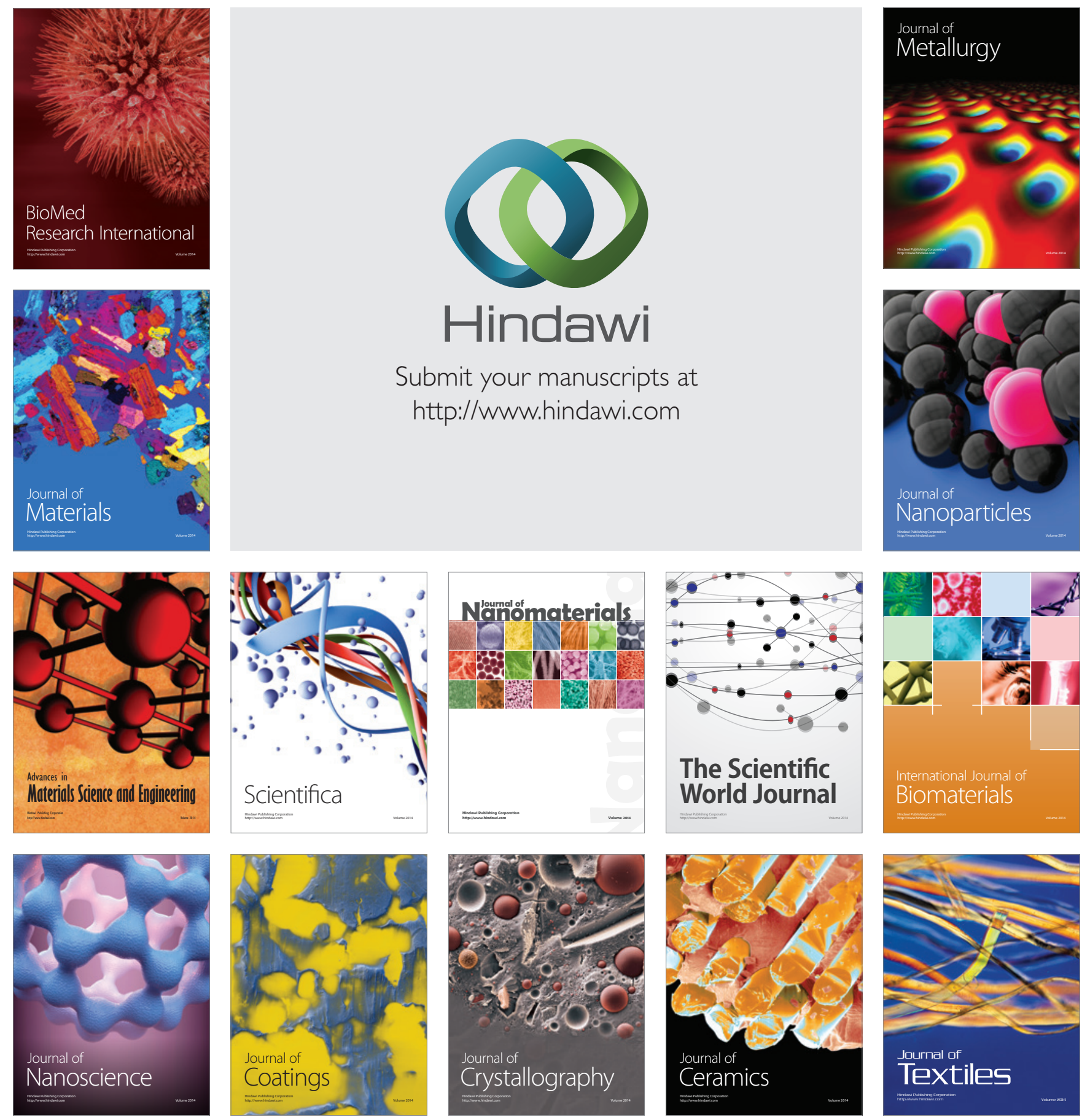